\title{
Ação pianística, aprendizagem motora e ciclos de movimento: uma análise cinematográfica bidimensional
}

\author{
Pianistic action, motor learning and cycles of
} movement: a two-dimensional cinematographic analysis

por Fernando Pabst Silva

\section{RESUMO}

Este artigo vem prestar conta dos resultados obtidos durante a realização do procedimento experimental previsto na pesquisa "Técnica, Movimento e Coordenação Motora - Conceitos e Aplicações Interdisciplinares na Ação Pianística". Baseando-se nos conhecimentos levantados e cruzados durante o decurso da pesquisa acerca das relações entre a ação pianística (PÓVOAS, 1999) e as ciências do movimento, 0 protocolo experimental foi informado e levado a cabo com sujeitos divididos entre dois grupos de sujeitos (GE e CC), utilizando-se a cinemetria como instrumento de coleta. Após a interpretação e disposição dos dados, os resultados obtidos trazem à tona conhecimentos relevantes no que tange a dependência entre biomecânica e ação pianística, assim como a necessidade de experimentos futuros a fim de refinar o perímetro de abrangência dos dados e sua reprodução.

Palavras-chave experimento biomecânico; ação pianística; coordenação motora; ciclos de movimento

\section{ABSTRACT}

This articles aims at presenting the data that resulted from a biomechanical experiment that was planned in the research project named "Technique, Movement and Motor Coordination - Concepts and Interdisciplinary Use in the Pianistic Action". Based on the information gathered and analyzed during the time of the research involving the pianistic action (PÓVOAS, 1999) and the sciences of human movement, an experimental protocol was written and executed with subjects being divided into two groups (Experiment and Control), by means of cinematographic analysis. By interpreting the resulting data, some conclusions relevant to the study of biomechanics and piano playing were drawn, as well as the need for finer experimental protocols to be executed in the future as to broaden the possibilities and reproducibility of these findings.

Keywords biomechanical experiment; pianistic action; motor coordination; cycles of movement 


\section{Introdução}

A pesquisa de onde origina este estudo, intitulada "Técnica, movimento e coordenação motora - conceitos e aplicações interdisciplinares na ação pianística", esmera-se em produzir conexões inter-áreas, a fim de lançar hipóteses de trabalho para o estudante e profissional do piano, através da discussão interdisciplinar possivel entre a ação pianística e as ciências do movimento. 0 grupo de pesquisadores encontra-se semanalmente e, por meio de levantamento bibliográfico, cruza informações de áreas como a biomecânica, ergonomia e cinesiologia com o estudo cotidiano ao instrumento, buscando inferir afinidades em potencial. Tendo em vista a importância da realização de um procedimento experimental com fins a consolidar e reforçar os dados obtidos durante as leituras, o grupo organizou e executou um experimento biomecânico, cujo protocolo e resultados são discutidos mais adiante, justificado pela conformação interdisciplinar da proposta original de pesquisa.

Compreende-se por ação pianística uma ação "construída através do processamento das questões envolvidas na música selecionando, coordenando e realizando tanto os elementos da construção musical que constituem e caracterizam cada obra quanto os movimentos que possibilitem esta ação" (PÓVOAS, 1999, p.80). Este entendimento dá clara ênfase ao tratamento biomecânico do ato pianístico, o que tem por objetivo a otimização, através do controle motor, do fazer ao piano. Um dos fatores que informam diretamente essa otimização é o aproveitamento axial desimpedido dos segmentos componentes do aparato pianístico, valorizando a fluência gestual.

A fluência gestual, que requer uma motricidade calculada, deve engajar todo o corpo e não somente os dedos (SILVA, 2010, p. 286), e merece, portanto, uma atenção deliberada (SLOBODA, 1996) por parte do instrumentista. Aqui entendidos meramente como terminais num complexo maior de jogos de alavancas, os dedos do pianista fazem "parte de um mecanismo que não pode funcionar com todas as suas vantagens inerentes sem o auxílio de um controle central" (WHITESIDE, 1997, p. 3). Cabe à área da aprendizagem motora e do desempenho motor postular acerca do funcionamento deste controle central, que competem mutuamente no decorrer da execução instrumental e também transversalmente, no decurso da vida do instrumentista.

Whiteside (1997, p. 6), pedagoga do instrumento, define que uma boa coordenação motora ao piano "teria [de utilizar] o princípio de que uma ação repetida por uma alavanca maior pode absorver ações de alavancas menores. A continuidade ativa das repetições das alavancas maiores não deve ser interrompida pelas ações das alavancas menores." A definição de uma hipótese que organizasse estes impulsos distintos (agonistas com antagonistas ao movimento pretendido) teria, portanto, como norte, também a economia e concatenação de impulsos de força.

A função organizacional, subentendida nos processos conscientes de aprendizagem motora, detém-se muito em aspectos cinesiológicos do controle motor, estes que produzem informações como "a posição das partes do corpo relativas umas às outras; a posição do corpo no espaço; os movimentos corporais; e a natureza dos obje- 
tos com os quais o corpo estabelece contato" (HAYWOOD; GETCHELL, 2004, p.187). Já a aprendizagem motora "é a conseqüência da co-adaptação entre o maquinário neural e a anatomia estrutural" (WOLPERT; GHAHRAMANI; FLANAGAN, 2001, p.488). Vale notar que "o sistema músculo-esquelético é altamente não-linear, no sentido de que somar duas seqüências de comandos motores não resulta na soma correspondente dos movimentos" (WOLPERT; GHAHRAMANI; FLANAGAN, 2001, p. 488), o que indica que um framework de estudo e ensino do instrumento deva considerar os riscos da atomização dos movimentos complexos em movimentos simples e não seqüenciados, o que, de acordo com Whiteside (1997, p.68), pode resultar num estudo lento pobre de antecipação e "responsável pela criação de uma infinidade de hábitos que serão prejudiciais para a obtenção de velocidade mais tarde".

A necessidade de se elaborar ferramentas teóricas que dêem conta da complexidade aqui disposta, ou seja, uma ferramenta que envolva os conhecimentos adquiridos das áreas biomecânica e ergonômica mostra-se como alternativa no momento atual da pedagogia do instrumento. Evitando dar continuidade a uma didática pianística que conte por definitivo certos estratagemas e paroxismos históricos, procuramos aqui propor uma situação de trabalho que responda ao chamado de interdisciplinaridade que tanto pode ajudar na busca de novas ideias. Lançamos uma hipótese de trabalho baseada nos ciclos de movimento (PÓVOAS, 1999).

Concebido como princípio de relação e regulação do impulso-movimento - ciclos de movimento - o recurso é assim definido pela autora:

[...] recurso técnico que prevê a organização do trabalho pianístico por meio da exploração consciente de movimentos nos eixos $x$, y e z e cuja flexibilização nestes eixos é orientada por linhas imaginárias ou desenhadas sobre trechos musicais de interesse. A opção pela linha de trajetória do ciclo é determinada pelo design musical, conforme a situação funcional mais eficiente, no sentido de otimizar a ação pianística. A concavidade ou convexidade do desenho das linhas indica a movimentação na profundidade da tecla ou coordenada z e a exploração deste eixo (z) induz à realização de movimentos menos retilíneos que permitem atingir o alvo (nota(s)) com um maior aproveitamento do impulso de um toque para o(s) próximo(s) e com maior segurança (PÓVOAS, 2007, p.544).

Esta opção intenciona, por meio de uma "racionalização e realização objetiva do movimento de acordo com o design musical, [diminuir] o somatório de distâncias a serem percorridas, e [resultar em] menos carga de trabalho com menor desgaste físico-muscular" (PÓVOAS, 1999, p. 90). A aplicação do recurso baseia-se nos benefícios pressupostos quando da utilização consciente e deliberada de sensações motoras, de economia de dispêndio energético, de uma maior significação da ação pianística que vem a trazer um maior apoio para o processo de aprendizado, além da realização otimizada do texto musical.

Um dos fundamentos do recurso, que é a flexibilização das trajetórias, conta com o fato de que movimentos retilíneos "são menos naturais quando comparados a 
um movimento complexo, operado por mais de uma alavanca, onde ocorre uma negociação de impulsos típica de um movimento experto (SILVA, 2010, p. 289)". Buscando refinar e atestar as hipóteses acima dispostas e comprovar a eficácia da utilização do recurso com estudantes de piano, o grupo elaborou o protocolo de um procedimento experimental, que encontra-se detalhado a seguir.

\section{Método}

Considerando, como foi discutido, que a execução musical, onde está situada a ação pianística, requer um igual comprometimento e ativação de Habilidades Cognitivo-Motoras e Capacidades Percepto-Motoras (MAGILL, 2000) equivalentes, um protocolo experimental foi traçado buscando provar a importância destes binômios. Tendo em mente os axiomas da área de coordenação motora que informam nosso arcabouço a nivel teórico, tais como: o posicionamento postural correto ao instrumento, a atenção aos eixos de movimentação, a preferência por gestos parabólicos sobre os retilíneos, uma pré-visão organizacional do movimento anterior à prática, uma coordenação negociada e vantajosa dos grupos musculares maiores com os menores e a ideia de que a soma de dois comandos motores não resulta num comando motor mais complexo devido ao caráter não-linear do sistema músculo-esquelético, encontra-se descrito a seguir o delineamento experimental desta pesquisa.

Um montante de 10 alunos, dentre os quais graduandos, mestrandos e mestres na área de piano da Universidade do Estado de Santa Catarina (UDESC) foi divido em dois grupos, 5 no Grupo Experimental (GE) e 5 no Grupo de Controle (CC). Os dois grupos receberam um trecho musical retirado da obra Lundu, de Camargo Guarnieri, a fim de que estudassem. Durante 10 encontros o Grupo Experimental recebeu, semanalmente, cerca de uma hora de treinamento do Grupo de Pesquisa (GP), composto por 3 pesquisadores, enquanto o Grupo Controle comprometeu-se em registrar as horas de estudo ao instrumento numa tabela providenciada pelo GP, podendo, de acordo com a vontade individual, buscar treinamento com outros professores. Ao final da etapa de treinamento, realizou-se o período de gravação e consequente interpretação dos dados.

Durante 0 treinamento, o Grupo Experimental recebeu instruções de como o estudo deveria ser realizado, além de exposições empíricas ao piano, onde cada sujeito teve diversas oportunidades de testar a utilização correta do recurso ciclos de movimento. 0 grupo disponibilizou material contendo a partitura acrescida de setas que informariam a direção e trajetória das parabolizações da mão, punho e antebraço (Figura 1). Estas setas foram confeccionadas baseadas no recurso ciclos de movimento (PÓVOAS, 1999):

A operacionalização deste princípio inicia com a decodificação analítica do design musical em termos de gestos, para os quais contribuem as noções de impulsão ou apoio inicial e apoio final ou queda. Distribuídas em três fases (apoio inicial, 
percurso e apoio final) as linhas imaginárias da [Figura 1] segmentam em impulsos temporários a ação pianística, a fim de que, quando aplicadas em seqüência, estas fases resultem num movimento abrangente e harmônico constituído de movimentos menores (discretos). A elaboração das linhas imaginárias leva em conta o resultado sonoro pretendido e o design musical subsequente, e [...] é senão uma adequação analítica dos membros superiores ao texto musical (SILVA; PÓVOAS, 2009, p. 4).

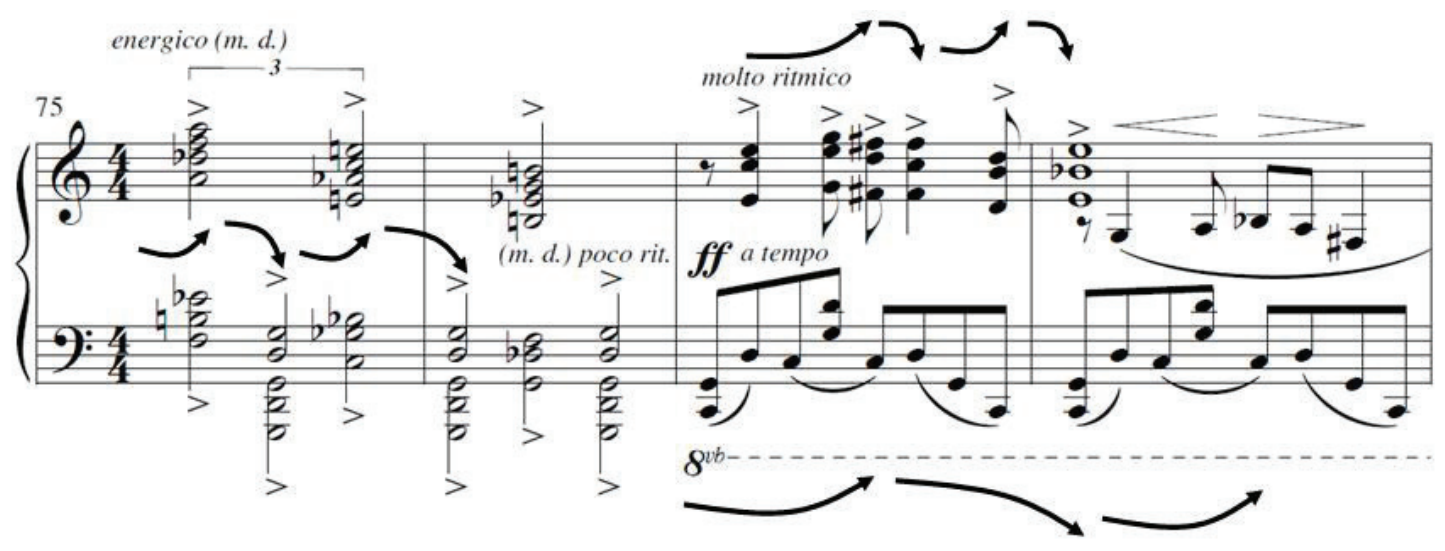

Figura 1. Sistema retirado do trecho musical entregue ao GE. A notação das linhas imaginária por vezes refere-se à coordenação bimanual, por vezes a um segmento específico. Fonte: Guarnieri: Lundu, cs $75-78$

Como métodos de coleta e análise de dados foram utilizados a cinemetria (ou cinematografia) e centrais inerciais espaciais. A cinemetria, ou cinematografia, registra "medições dos movimentos realizados pelo(s) executante(s) através de imagens, registro de trajetórias, decurso de tempo, determinação de curvas de velocidade e de aceleração, entre outras variáveis" (SILVA; PÓVOAS, 2008, p. 5-6). Para a captação cinemática, comumente utilizam-se aparelhos videográficos de alta velocidade e rendimento, o que, em conjunto com a espacialização de determinados segmentos através de pontos luminosos, pode gerar modelos matemáticos em função dos movimentos captados. Estes pontos luminosos, no caso do presente protocolo, foram situados (1) no antebraço esquerdo, próximo ao cotovelo, (2) na mão esquerda, (3) no antebraço direito e (4) na mão direita, centralizados.

As centrais inerciais (4) foram distribuídas de maneira idêntica à dos pontos luminosos. No entanto, seu funcionamento difere ao da cinemetria: dispensam o uso de videografia e captam, em tempo real, o posicionamento no espaço dos segmentos aonde são fixadas. Depreendem-se também a aceleração, variações angulares, velocidade e relação com as outras centrais, de forma supervisionada por um controlador central que recebe os dados via radiofreqüência no decorrer do registro. Um computador foi utilizado como controlador central, e armazenou uma imagem digital de três dimensões para cada $\mathrm{Cl}$ afixada.

A utilização destes modos de análise permite inferir certas características matemáticas e a estrutura de determinados movimentos que são descritos na seção 
seguinte. Na Figura 2 temos em diagrama as especificidades acima descritas da montagem experimental, do ponto de vista da câmera de alta velocidade usada para a captação bidimensional.

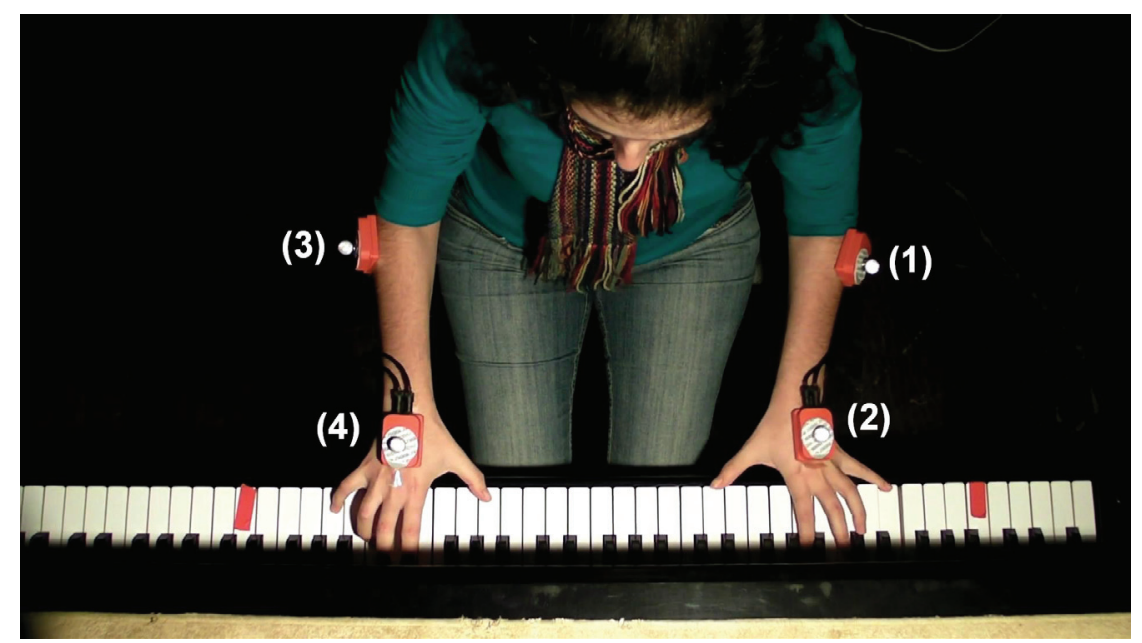

Figura 2. Disposição dos pontos luminosos (marcadores), fixados em cima das centrais inerciais $(\mathrm{Cl})$

\section{Resultados e discussão}

Durante a captação, todos os sujeitos gravaram o trecho completo que foi entregue aos grupos (do compasso 75 até o início do 88), do começo ao fim, sem interrupções, com direito a três tentativas cada um. Para o caso do presente artigo, foi selecionada para análise a parte correspondente aos compassos 77 e 78 (Figura 1), considerando-se os pontos luminosos referentes ao segmento mão esquerda (marcador 2 da Figura 2). Como coleta, foi feito uso da gravação cinematográfica de uma câmera somente que produziu, portanto, uma imagem bidimensional dos eixos $X$ e $Y$ do movimento. As imagens coletadas mais tarde foram digitalizadas utilizando o software de análise de movimento DgeeMe v1.0 e os dados, posteriormente, foram tabelados e confrontados nas suas grandezas. A análise tridimensional de todos os pontos (eixos $X, Y$ e $Z$ ) resta ser analisada e interpretada futuramente.

Dos dados obtidos, foram traçados dois gráficos de densidade (Figura 3) que representam, nas regiões mais densas, uma maior coincidência de trajeto entre os diversos sujeitos de determinado grupo e, nas menos densas, uma menor incidência, caracterizando como média do grupo os números obtidos. A perspectiva é a de trajetória no espaço delimitado em metros, com o eixo $X$ correspondendo à linha paralela ao teclado e o eixo $\mathrm{Y}$, à linha perpendicular ao teclado. 

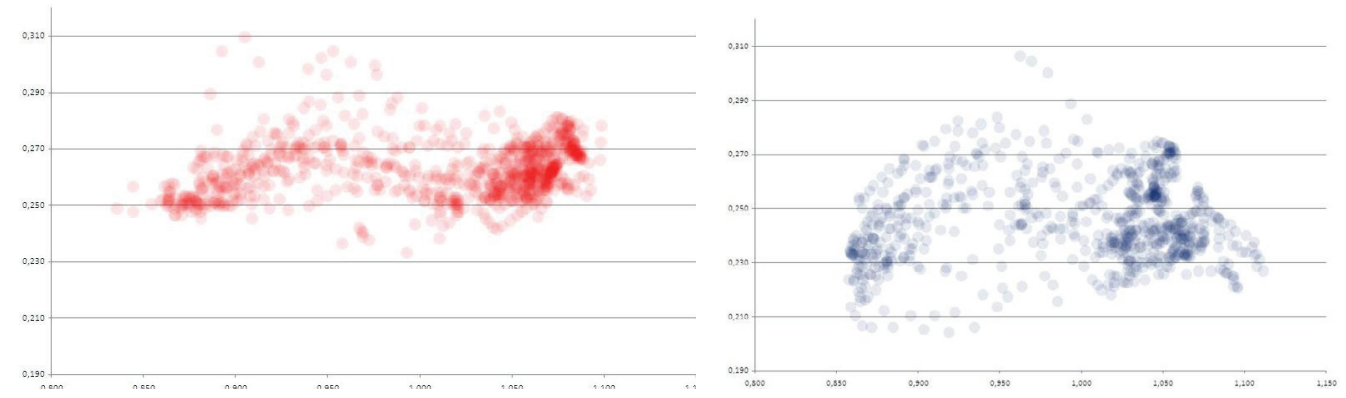

Figura 3. Gráfico de dispersão espacial do GC (à esquerda) e do GE (à direita), representando maior ou menor incidência de trajetória comum entre os sujeitos de um determinado grupo, a título de média

Apesar de ser possível determinar que ambos os gráficos observam um certo padrão de distribuição, nota-se uma tendência no Grupo Experimental de maior aproveitamento do eixo Y em relação ao Grupo de Controle. Para confirmar esta tendência, o seguinte gráfico foi programado (Figura 4), onde o eixo $X$ representa 0 número de quadros (imagens produzidas pelo cinematógrafo) e o eixo $Y$ representa a variação de trajetória em metros realizada pelo marcador 2 (mão esquerda) em grandezas absolutas.

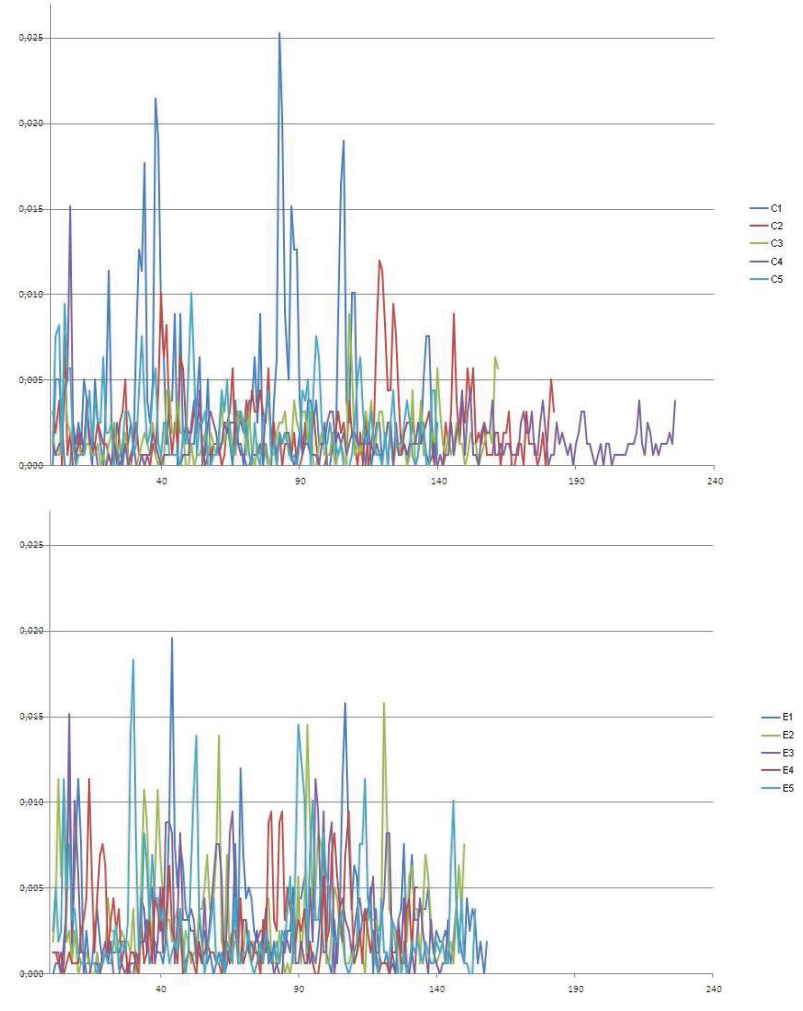

Figura 4. Gráfico representando variação de trajetória no eixo Y da mão esquerda para o GC, acima, e para o GE, abaixo

Os gráficos da Figura 4 confirmam a tendência observada nos gráficos da Figura 3 , mostrando que o GE teve, predominantemente, maior amplitude, visto maior variação, no eixo perpendicular ( $Y$ ) ao teclado. Uma possível interpretação destes dados 
nos leva a crer que, por consequência do treinamento recebido pelo $\mathrm{CE}$, o qual não foi realizado com o GC, durante a execução do trecho determinado os pianistas do Grupo Experimental realizaram maiores flexões do punho esquerdo, o que acarretou maior movimentação do marcador 2 e, portanto, uma soma maior de trajetória neste eixo. Adicionando-se a trajetória do marcador 2 de todos os sujeitos de um determinado grupo, teremos 2,128 metros para o GE, enquanto que somente 1,828 metros para o GC, caracterizando assim uma diferença razoável e relevante para as conclusões do experimento.

É possível lançar algumas hipóteses paras os dados obtidos. Primeiramente, a de que as parabolizações treinadas e desenhadas por meio do recurso ciclos de movimento durante as 10 sessões de treinamento de GE tenham ajudado na flexibilização deste eixo, haja vista que conceitos como a globalização do aparato pianístico fazem parte de sua proposta. Segundo, a de que o eixo $Y$ esteja captando ruídos da movimentação do punho no eixo Z (não gravado por cinemetria), o que resulta necessariamente na flexão da articulação punho-mão, o que por sua vez confere maior variação ao marcador 2 no eixo $Y$ - fato também previsto quando do treinamento, pois um maior aproveitamento no eixo de profundidade ( $Z$ ) foi um norte da aplicação dos ciclos de movimento.

Estes achados implicam, no entender do grupo de pesquisa, em algumas proposições: (a) de que o GE teve melhor rendimento do tempo de estudo, visto que sua gravação atingiu um nível mais veloz, com menor número de quadros por sujeito, fato este previsto no protocolo experimental, pois os ciclos de movimento permitem desenvolver uma maior velocidade de execução (rapidez de movimento) devido à otimização da trajetória dentro de cada ciclo de movimento (PÓVOAS, 2007); (b) de que o engajamento mais consciente e ativo das diversas articulações dos membros superiores teve um benéfico efeito cognitivo-motor ao nível de aprendizado e retenção de aprendizado, pois "a compreensão e a elaboração exatas das informações sensoriais de movimento como base de uma direção e regulação corretas do decurso de movimento [são] processo essencial da coordenação motora" (MEINEL, 1987, p. 153); e (c) de que a liberdade axial de movimentos observada no GE poupou de maneira mais saudável as articulações devido ao estímulo à flexibilidade, "sinônimo de amplitude do movimento articular" (RASCH, 1991, p. 18-19), estratégia esta muito benéfica a longo prazo, já que visa "manter a higidez e a integridade das estruturas anatômicas ativas durante a prática pianística" (PÓVOAS; PONTES; SILVA, 2012, p. 2).

Os dados advindos da coleta tridimensional deverão, no futuro, ser analisados de modo a emprestar maior credibilidade a estes achados. A possibilidade, porém, da contradição destes resultados é mantida patente e encarada como frutífera e produtiva, visto que agregará mais informação para a escrita de novos protocolos experimentais de pesquisa. A perseguição de melhor e mais finos dados é uma busca prevista e inextinguível da pesquisa atual, e planeja-se a utilização, nos próximos experimentos, de um método qualitativo de análise para as gravações em áudio dos sujeitos. 


\section{Conclusão}

Como escreve Kaplan (1987, p. 45) “do ponto de vista da execução instrumental, a aquisição e posterior reorganização dos hábitos constitui a base sobre a qual irá se construir a técnica". Buscar subsídios para melhor definir e realizar esta organização foi um dos objetivos deste artigo. Intentou-se, através da utilização do princípio de ação contido no recurso ciclos de movimento, confirmar ou não suposições acerca dos benefícios propostos na formulação de sua problemática, que incluem um melhor rendimento energético, uma maior consciência corporal, e um menor desgaste articular, entre outros, no instante da execução sonora.

No que tange a abrangência do experimento realizado, Magill (2000, p. 137) também nos lembra que o "processo de aprendizagem pode ser observado de acordo com quatro características gerais do desempenho, que são: aperfeiçoamento, consistência, persistência e adaptabilidade". A adaptabilidade mencionada seria a capacidade de transporte, o recurso de poder utilizar uma habilidade específica, aprendida num certo contexto, extrapolando-se a situação original. 0 aporte de um repertório específico de gestos adequados a situações técnicas específicas e a elaboração um recurso que dê conta desta tarefa complexa, mantendo-se sempre em discussão e sendo, portanto, de uma plasticidade funcional, não deixa de ser um dos objetivos da pesquisa que resultou neste experimento.

Vale notar que os dados presentes neste artigo não se encontram extinguidos e podem passar por futura reinterpretação no momento da análise dos dados restantes, assim como o segmento direito, outras seções da peça, ou as informações tridimensionais acumuladas pelas centrais inerciais. Não se descarta a necessidade da realização de novos experimentos biomecânicos pelo grupo no futuro, objetivando um aporte maior de dados que venham a comprovar mais firmemente as hipóteses da pesquisa, sempre levando em conta a prioridade da sustentação científica das generalizações implicadas na utilização do formato Grupo Experimental e Grupo de Controle (BARROS, 2010).

À luz do que foi relatado acima, pretende-se que os enunciados de áreas como biomecânica, cinesiologia, ergonomia e sub-áreas como controle e aprendizado motor façam cada vez mais parte do cotidiano do estudante e professor de piano. A contribuição em potencial destas áreas ditas alheias pode vir a informar novos conceitos e modos de trabalho que sejam mais condizentes com o contexto atual da produção de conhecimento científico, que procura democratizar e desmistificar tabus produzidos pela historicamente precária transmissão de conhecimentos no ensino do piano. 


\section{Referências bibliográficas}

> BARROS, L. C. As pesquisas experimentais e pesquisas descritivas com delineamento experimental na área de práticas interpretativas: Considerações sobre o Grupo de Controle (GC) e Grupo Experimental (GE). In.: Anais do XX Congresso da ANPPOM, Florianópolis. Centro de Artes da Universidade do Estado de Santa Catarina, 2010. p. 1265-1269.

> HAYWOOD, K.; GETCHELL, N. Desenvolvimento motor ao longo da vida. 3.ed. Porto Alegre: Artmed, 2004.

> KAPLAN, J. Teoria da Aprendizagem Pianística. Porto Alegre: Movimento, 1987.

> MAGILL, R. Aprendizagem Motora: Conceitos e Aplicações. São Paulo: Edgard Blücher, 2000.

> MEINEL, C. Motricidade I: Teoria da Motricidade Esportiva sob o Aspecto Pedagógico. São Paulo: Ao Livro Técnico, 1987.

> PóvOAS, M. B. C. Controle de Movimento com Base em um Princípio de Relação e Regulação do Impulso-Movimento: Possíveis Reflexos na Otimização da Ação Pianística. Tese de Doutorado. UFRGS, Porto Alegre, 1999.

$>$ Ação pianística, desempenho e controle do movimento: uma perspectiva interdisciplinar.

> In: Anais do III Simpósio de Cognição e Artes Musicais. Salvador: EDUFBA, 2007. p. 540-548.

> PÓVOAS, M. B. C.; PONTES, V. E.; SILVA, F. P. Conexões teóricas e práticas entre 0 fator do desempenho flexibilidade e a ação pianística. Um panorama interdisciplinar. In.: Anais do XXII Congresso da ANPPOM, João Pessoa. UFPB, 2012. No prelo.

> PóvOAS, M. B. C.; SILVA, D. Métodos de análise do movimento: Aplicações na ação pianística. Revista DAPesquisa. Florianópolis: Centro de Artes, UDESC. Vol. 3 ก. 1, 2008.

> PÓVOAS, M. B. C.; SILVA, F. P. Ação pianística e interdisciplinaridade: aplicando conceitos de coordenação e aprendizagem motora ao piano. Revista DAPesquisa. Florianópolis: Centro de Artes, UDESC. Vol. 3 n. 2, 2009.

> RASCH, P. Cinesiologia e anatomia aplicada. Rio de Janeiro: Guanabara Koogan, 1991.

> SILVA, F. P. Aprendizagem e Desempenho Motor: Esboçando uma didática pianística a partir dos ciclos de movimento. Revista DAPesquisa. Florianópolis: Centro de Artes, UDESC. n.7, 2010, p. 284-293.

> SLOBODA, J. The acquisition of musical performance expertise: deconstructing the "talent" account of individual differences in musical expressivity. In: K.A. ERICSSON (Ed.), The Road to excellence: the acquisition of expert performance in arts and sciences, sports and games. New Jersey: Lawrence Erlbaum, 1996, p. 107-126. 
> WHITESIDE, A. On Piano Playing. Portland, Oregon: Amadeus Press, 1997.

$>$ WOLPERT, D.; GHAHRAMANI, Z.; FLANAGAN, J. Perspectives and problems in motor learning. In: Trends in Cognitive Sciences, Vol.5 No.11. November 2001. p. 487-494.

Fernando Pabst Silva, graduando do curso de Bacharelado em Piano, CEART-UDESC fernandopabst@gmail.com 